\title{
Hyers-Ulam and Hyers-Ulam-Rassias stability of a class of Hammerstein integral
}

equations

L. P. Castro and A. M. Simões

Citation: 1798, 020036 (2017); doi: 10.1063/1.4972628

View online: http://dx.doi.org/10.1063/1.4972628

View Table of Contents: http://aip.scitation.org/toc/apc/1798/1

Published by the American Institute of Physics 


\title{
Hyers-Ulam and Hyers-Ulam-Rassias Stability of a Class of Hammerstein Integral Equations
}

\author{
L. P. Castro ${ }^{1, a), b)}$ and A. M. Simões ${ }^{2, c)}$ \\ ${ }^{1}$ CIDMA - Center for Research and Development in Mathematics and Applications, Department of Mathematics, \\ University of Aveiro, 3810-193 Aveiro, Portugal. \\ ${ }^{2}$ CMA-UBI - Center of Mathematics and Applications of University of Beira Interior, Department of Mathematics, \\ University of Beira Interior, 6200-001 Covilhã, Portugal.

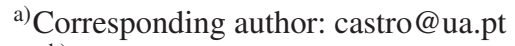 \\ b) URL: http://sweet.ua.pt/castro/ \\ c)asimoes@ubi.pt
}

\begin{abstract}
The purpose of this paper is to study different kinds of stability for a class of Hammerstein integral equations. Sufficient conditions are derived in view to obtain Hyers-Ulam stability and Hyers-Ulam-Rassias stability for such a class of Hammerstein integral equations. The consequent different cases of a finite interval and an infinite interval are considered, and some concrete examples are included to illustrate the results.
\end{abstract}

\section{INTRODUCTION}

During the last six decades the concept of stability for different kinds of equations has been studied in a quite extensive way. In special, Hyers-Ulam stability and Hyers-Ulam-Rassias stability of a great variety of functional equations, differential equations and integral equations have earned particular interest due to their great number of applications e.g. in elasticity, semiconductors, heat conduction, fluid flow, scattering theory, chemical reactions and population dynamic, among others (see $[1,3,4,5,6,7,9,10,11,12,14,15,16,17,18,19,20,21,23]$ ). The first results of stability of this type for functional equations were originated from a famous question raised by S. M. Ulam, in 1940, about to discover when a solution of an equation differing "slightly" from a given one must be somehow near to the solution of the given equation.

D. H. Hyers gave a partial answer to the question of S. M. Ulam, for Banach spaces, in the case of the additive Cauchy equation, $f(x+y)=f(x)+f(y)$, cf. [13]. Namely, let $S_{1}$ and $S_{2}$ be two (real) Banach spaces and assume that a mapping $h: S_{1} \rightarrow S_{2}$ satisfies the inequality

$$
\|h(x+y)-h(x)-h(y)\| \leq \varepsilon, \quad x, y \in S_{1}
$$

for some nonnegative $\varepsilon$. Then the limit $\mathcal{A}(x)=\lim _{n \rightarrow \infty} h\left(2^{n} x\right) 2^{-n}$ exists for each $x \in S_{1}$ and $\mathcal{A}: S_{1} \rightarrow S_{2}$ is the unique additive function such that

$$
\|\mathcal{A}(x)-h(x)\| \leq \varepsilon, \quad x \in S_{1}
$$

holds. This last result is nowadays called the Hyers-Ulam stability of the additive Cauchy equation.

New directions were introduced by Th. M. Rassias, see [22], by considering unbounded right-hand sides in the inequality (1), which depend on certain functions of $x$ and $y$, introducing therefore the so-called Hyers-Ulam-Rassias stability.

In this work, we will be devoted to analyse Hyers-Ulam and Hyers-Ulam-Rassias stability for the following class of Hammerstein integral equations:

$$
y(x)=p(x)+f(x, y(x)) \int_{a}^{x} g(x, \tau) h(\tau, y(\tau)) d \tau, \quad x \in[a, b],
$$


where, for starting, $a$ and $b$ are fixed real numbers, $p:[a, b] \rightarrow \mathbb{C}, f:[a, b] \times \mathbb{C} \rightarrow \mathbb{C}$ and $h:[a, b] \times \mathbb{C} \rightarrow \mathbb{C}$ are continuous functions, and the kernel $g:[a, b] \times[a, b] \rightarrow \mathbb{C}$ is also a continuous function.

The formal definition of the above mentioned Hyers-Ulam-Rassias stability and Hyers-Ulam stability are now introduced for the Hammerstein integral equation which are being considered in here.

If for each function $y$ satisfying

$$
\left|y(x)-p(x)-f(x, y(x)) \int_{a}^{x} g(x, \tau) h(\tau, y(\tau)) d \tau\right| \leq \sigma(x), \quad x \in[a, b]
$$

where $\sigma$ is a non-negative function, there is a solution $y_{0}$ of the Hammerstein integral equation and a constant $C>0$ independent of $y$ and $y_{0}$ such that

$$
\left|y(x)-y_{0}(x)\right| \leq C \sigma(x)
$$

for all $x \in[a, b]$, then we say that the Hammerstein integral equation (2) has the Hyers-Ulam-Rassias stability.

If for each function $y$ satisfying

$$
\left|y(x)-p(x)-f(x, y(x)) \int_{a}^{x} g(x, \tau) h(\tau, y(\tau)) d \tau\right| \leq \theta, \quad x \in[a, b]
$$

where $\theta \geq 0$, there is a solution $y_{0}$ of the Hammerstein integral equation and a constant $C>0$ independent of $y$ and $y_{0}$ such that

$$
\left|y(x)-y_{0}(x)\right| \leq C \theta
$$

for all $x \in[a, b]$, then we say that the Hammerstein integral equation has the Hyers-Ulam stability.

Some of the present techniques to study the stability of functional equations use a combination of fixed point results with a generalized metric in appropriate settings. In view of this, let us recall the definition of a generalized metric on a nonempty set $X$.

Definition 1 A function $d: X \times X \rightarrow[0,+\infty]$ is called a generalized metric on $X$ if and only if $d$ satisfies the following three propositions:

i) $d(x, y)=0$ if and only if $x=y$;

ii) $d(x, y)=d(y, x)$ for all $x, y \in X$;

iii) $d(x, z) \leq d(x, y)+d(y, z)$ for all $x, y, z \in X$.

In the just presented setting of generalized metrics, it is possible to use the following well-known Banach Fixed Point Theorem.

Theorem 2 Let $(X, d)$ be a generalized complete metric space and $T: X \rightarrow X$ a strictly contractive operator with a Lipschitz constant $L<1$. If there exists a nonnegative integer $k$ such that $d\left(T^{k+1} x, T^{k} x\right)<\infty$ for some $x \in X$, then the following three propositions hold true:

i) the sequence $\left(T^{n} x\right)_{n \in \mathbb{N}}$ converges to a fixed point $x^{*}$ of $T$;

ii) $x^{*}$ is the unique fixed point of $T$ in

$$
X^{*}=\left\{y \in X: d\left(T^{k} x, y\right)<\infty\right\}
$$

iii) if $y \in X^{*}$, then

$$
d\left(y, x^{*}\right) \leq \frac{1}{1-L} d(T y, y) .
$$




\section{THE HYERS-ULAM-RASSIAS STABILITY IN THE FINITE INTERVAL CASE}

The present section is devoted to present sufficient conditions for the Hyers-Ulam-Rassias stability of the Hammerstein integral equation (2), where $x \in[a, b]$, for some fixed real numbers $a$ and $b$.

We will be using the space $C([a, b])$ of continuous functions on $[a, b]$, endowed with the metric

$$
d(u, v)=\sup _{x \in[a, b]} \frac{|u(x)-v(x)|}{\sigma(x)},
$$

where $\sigma$ is a non-decreasing continuous function $\sigma:[a, b] \rightarrow(0, \infty)$. Recall that $(C([a, b]), d)$ is a complete metric space (cf., e.g., [2], [8]).

Theorem 3 Let us consider a continuous given function $\mu:[a, b] \rightarrow[0, \infty)$. Moreover, assume that $p:[a, b] \rightarrow \mathbb{C}$ is a continuous function, $f:[a, b] \times \mathbb{C} \rightarrow \mathbb{C}$ is a continuous function such that there exists $M>0$ so that

$$
M=\sup _{x \in[a, b]}|f(x, y)|, \quad y \in \mathbb{C},
$$

and the kernel $g:[a, b] \times[a, b] \rightarrow \mathbb{C}$ is also continuous. In addition, suppose that there is $\beta \in[0,1)$ such that

$$
\int_{a}^{x}|g(x, \tau)| \mu(\tau) \sigma(\tau) d \tau \leq \beta \sigma(x)
$$

and $h:[a, b] \times \mathbb{C} \rightarrow \mathbb{C}$ is a continuous function which fulfils the condition

$$
|h(x, u(x))-h(x, v(x))| \leq \mu(x)|u(x)-v(x)|
$$

for all $x \in[a, b]$ and $u, v \in C([a, b])$.

If $y \in C([a, b])$ is such that

$$
\left|y(x)-p(x)-f(x, y(x)) \int_{a}^{x} g(x, \tau) h(\tau, y(\tau)) d \tau\right| \leq \sigma(x), \quad x \in[a, b],
$$

and $M \beta<1$, then there is a unique function $y_{0} \in C([a, b])$ such that

$$
y_{0}(x)=p(x)+f\left(x, y_{0}(x)\right) \int_{a}^{x} g(x, \tau) h\left(\tau, y_{0}(\tau)\right) d \tau
$$

and

$$
\left|y(x)-y_{0}(x)\right| \leq \frac{\sigma(x)}{1-M \beta}
$$

for all $x \in[a, b]$.

This means that under the above conditions, the Hammerstein integral equation (2) has the Hyers-Ulam-Rassias stability.

Proof. We will consider the operator $T: C([a, b]) \rightarrow C([a, b])$, defined by

$$
(T u)(x)=p(x)+f(x, u(x)) \int_{a}^{x} g(x, \tau) h(\tau, u(\tau)) d \tau,
$$

for all $x \in[a, b]$ and $u \in C([a, b])$. Note that for any continuous function $u, T u$ is also continuous. Indeed,

$$
\begin{aligned}
\left|(T u)(x)-(T u)\left(x_{0}\right)\right| & =\left|p(x)+f(x, u(x)) \int_{a}^{x} g(x, \tau) h(\tau, u(\tau)) d \tau-p\left(x_{0}\right)-f\left(x_{0}, u\left(x_{0}\right)\right) \int_{a}^{x_{0}} g\left(x_{0}, \tau\right) h(\tau, u(\tau)) d \tau\right| \\
& \leq\left|p(x)-p\left(x_{0}\right)\right|+\left|f(x, u(x)) \int_{a}^{x} g(x, \tau) h(\tau, u(\tau)) d \tau-f\left(x_{0}, u\left(x_{0}\right)\right) \int_{a}^{x_{0}} g\left(x_{0}, \tau\right) h(\tau, u(\tau)) d \tau\right|
\end{aligned}
$$




$$
\begin{gathered}
\leq\left|p(x)-p\left(x_{0}\right)\right|+M\left|\int_{a}^{x} g(x, \tau) h(\tau, u(\tau)) d \tau-\int_{a}^{x_{0}} g\left(x_{0}, \tau\right) h(\tau, u(\tau)) d \tau\right| \\
=\left|p(x)-p\left(x_{0}\right)\right|+M \mid \int_{a}^{x} g(x, \tau) h(\tau, u(\tau)) d \tau-\int_{a}^{x} g\left(x_{0}, \tau\right) h(\tau, u(\tau)) d \tau \\
\quad+\int_{a}^{x} g\left(x_{0}, \tau\right) h(\tau, u(\tau)) d \tau-\int_{a}^{x_{0}} g\left(x_{0}, \tau\right) h(\tau, u(\tau)) d \tau \mid \\
\leq\left|p(x)-p\left(x_{0}\right)\right|+M\left(\int_{a}^{x}\left|g(x, \tau)-g\left(x_{0}, \tau\right)\right||h(\tau, u(\tau))| d \tau\right. \\
\left.+\left|\int_{x_{0}}^{x} g\left(x_{0}, \tau\right) h(\tau, u(\tau)) d \tau\right|\right) \rightarrow 0
\end{gathered}
$$

when $x \rightarrow x_{0}$.

Under the present conditions, we will now deduce that the operator $T$ is strictly contractive (with respect to the metric under consideration). Indeed, for all $u, v \in C([a, b])$, we have,

$$
\begin{aligned}
d(T u, T v) & =\sup _{x \in[a, b]} \frac{|(T u)(x)-(T v)(x)|}{\sigma(x)} \\
& =\sup _{x \in[a, b]} \frac{\left|f(x, u(x)) \int_{a}^{x} g(x, \tau) h(\tau, u(\tau)) d \tau-f(x, v(x)) \int_{a}^{x} g(x, \tau) h(\tau, v(\tau)) d \tau\right|}{\sigma(x)} \\
& \leq M \sup _{x \in[a, b]} \frac{\int_{a}^{x}|g(x, \tau)||h(\tau, u(\tau))-h(\tau, v(\tau))| d \tau}{\sigma(x)} \\
& M \sup _{x \in[a, b]} \frac{\int_{a}^{x}|g(x, \tau)| \mu(\tau)|u(\tau)-v(\tau)| d \tau}{\sigma(x)} \\
& =M \sup _{x \in[a, b]} \frac{\int_{a}^{x}|g(x, \tau)| \mu(\tau) \sigma(\tau) \frac{|u(\tau)-v(\tau)|}{\sigma(\tau)} d \tau}{\sigma(x)} \\
& \leq M \sup _{\tau \in[a, b]} \frac{|u(\tau)-v(\tau)|}{\sigma(\tau)} \sup _{x \in[a, b]} \frac{\int_{a}^{x}|g(x, \tau)| \mu(\tau) \sigma(\tau) d \tau}{\sigma(x)} \\
& \leq M d(u, v) \beta .
\end{aligned}
$$

Due to the fact that $M \beta<1$ it follows that $T$ is strictly contractive. Thus, we can apply the above mentioned Banach Fixed Point Theorem, which ensures that we have the Hyers-Ulam-Rassias stability for the Hammerstein integral equation. Additionally, (6) follows from (3) and (5).

\section{THE HYERS-ULAM STABILITY IN THE FINITE INTERVAL CASE}

The present section is devoted to present sufficient conditions for the Hyers-Ulam stability of the Hammerstein integral equation (2). For a given non-decreasing continuous function $\sigma:[a, b] \rightarrow(0, \infty)$, we will continue to use the same metric (4).

Theorem 4 Let us consider a continuous given function $\mu:[a, b] \rightarrow[0, \infty)$. Moreover, assume that $p:[a, b] \rightarrow \mathbb{C}$ is a continuous function, $f:[a, b] \times \mathbb{C} \rightarrow \mathbb{C}$ is a continuous function such that there exists $M>0$ so that

$$
M=\sup _{x \in[a, b]}|f(x, y)|, \quad y \in \mathbb{C},
$$

and the kernel $g:[a, b] \times[a, b] \rightarrow \mathbb{C}$ is also continuous. In addition, suppose that there is $\beta \in[0,1)$ such that

$$
\int_{a}^{x}|g(x, \tau)| \mu(\tau) \sigma(\tau) d \tau \leq \beta \sigma(x)
$$


and $h:[a, b] \times \mathbb{C} \rightarrow \mathbb{C}$ is a continuous function which fulfils the condition

$$
|h(x, u(x))-h(x, v(x))| \leq \mu(x)|u(x)-v(x)|
$$

for all $x \in[a, b]$ and $u, v \in C([a, b])$.

If $y \in C([a, b])$ is such that

$$
\left|y(x)-p(x)-f(x, y(x)) \int_{a}^{x} g(x, \tau) h(\tau, y(\tau)) d \tau\right| \leq \theta, \quad x \in[a, b],
$$

where $\theta \geq 0$ and $M \beta<1$, then there is a unique function $y_{0} \in C([a, b])$ such that

$$
y_{0}(x)=p(x)+f\left(x, y_{0}(x)\right) \int_{a}^{x} g(x, \tau) h\left(\tau, y_{0}(\tau)\right) d \tau
$$

and

$$
\left|y(x)-y_{0}(x)\right| \leq \frac{\theta}{1-M \beta}
$$

for all $x \in[a, b]$.

This means that under the above conditions, the Hammerstein integral equation (2) has the Hyers-Ulam stability.

Proof. We will consider the operator $T: C([a, b]) \rightarrow C([a, b])$, defined by

$$
(T u)(x)=p(x)+f(x, u(x)) \int_{a}^{x} g(x, \tau) h(\tau, u(\tau)) d \tau,
$$

for all $x \in[a, b]$ and $u \in C([a, b])$ (which we know already to be well-defined).

$T$ is strictly contractive (with respect to the metric under consideration). Indeed, for all $u, v \in C([a, b])$, we have,

$$
\begin{aligned}
d(T u, T v) & =\sup _{x \in[a, b]} \frac{|(T u)(x)-(T v)(x)|}{\sigma(x)} \\
& \leq M \sup _{x \in[a, b]} \frac{\int_{a}^{x}|g(x, \tau)||h(\tau, u(\tau))-h(\tau, v(\tau))| d \tau}{\sigma(x)} \\
& \leq M \sup _{x \in[a, b]} \frac{\int_{a}^{x}|g(x, \tau)| \mu(\tau)|u(\tau)-v(\tau)| d \tau}{\sigma(x)} \\
& =M \sup _{x \in[a, b]} \frac{\int_{a}^{x}|g(x, \tau)| \mu(\tau) \sigma(\tau) \frac{|u(\tau)-v(\tau)|}{\sigma(\tau)} d \tau}{\sigma(x)} \\
& \leq M \sup _{\tau \in[a, b]} \frac{|u(\tau)-v(\tau)|}{\sigma(\tau)} \sup _{x \in[a, b]} \frac{\int_{a}^{x}|g(x, \tau)| \mu(\tau) \sigma(\tau) d \tau}{\sigma(x)} \\
& \leq M d(u, v) \beta .
\end{aligned}
$$

Due to the fact that $M \beta<1$ it follows that $T$ is strictly contractive. Thus, we can again apply the Banach Fixed Point Theorem, which ensures that we have the Hyers-Ulam stability for the Hammerstein integral equation with (8) being obtained by using (3) and (7).

\section{THE HYERS-ULAM-RASSIAS STABILITY IN THE INFINITE INTERVAL CASE}

In this section, we will analyse the Hyers-Ulam-Rassias stability of the Hammerstein integral equation but when considering infinite intervals. This means that instead of considering, as before, a finite interval $[a, b]$ (with $a, b \in \mathbb{R}$ ), we will now consider e.g. corresponding intervals $[a, \infty)$, for some fixed $a \in \mathbb{R}$. 
Thus, we will now be dealing with the integral equation

$$
y(x)=p(x)+f(x, y(x)) \int_{a}^{x} g(x, \tau) h(\tau, y(\tau)) d \tau, \quad x \in[a, \infty),
$$

where $a$ is a fixed real number, $p:[a, \infty) \rightarrow \mathbb{C}$ and $f:[a, \infty) \times \mathbb{C} \rightarrow \mathbb{C}$ are bounded continuous functions, and $h:[a, \infty) \times \mathbb{C} \rightarrow \mathbb{C}$ and the kernel $g:[a, \infty) \times[a, \infty) \rightarrow \mathbb{C}$ are continuous functions. Here, our strategy will be based on a recurrence procedure due to the already obtained result for the corresponding finite interval case.

Let us consider a fixed non-decreasing continuous function $\varphi:[a, \infty) \rightarrow(\varepsilon, \omega)$, for some $\varepsilon, \omega>0$, and the space $C_{b}([a, \infty))$ of bounded continuous functions endowed with the weighted metric

$$
d_{b}(u, v)=\sup _{x \in[a, \infty)} \frac{|u(x)-v(x)|}{\varphi(x)} .
$$

Theorem 5 Let us consider a continuous given function $\mu:[a, \infty) \rightarrow[0, \infty)$. Moreover, assume that $p:[a, \infty) \rightarrow$ $\mathbb{C}$ is a bounded continuous function, $f:[a, \infty) \times \mathbb{C} \rightarrow \mathbb{C}$ is a continuous function such that there exists $M>0$ so that

$$
M=\sup _{x \in[a, \infty)}|f(x, y)|, \quad y \in \mathbb{C},
$$

and the kernel $g:[a, \infty) \times[a, \infty) \rightarrow \mathbb{C}$ and $h:[a, \infty) \times \mathbb{C} \rightarrow \mathbb{C}$ are continuous functions so that $\int_{a}^{x} g(x, \tau) h(\tau, z(\tau)) d \tau$ is a bounded continuous function for any bounded continuous function $z$.

In addition, suppose that there is $\beta \in[0,1)$ such that

$$
\int_{a}^{x}|g(x, \tau)| \mu(\tau) \varphi(\tau) d \tau \leq \beta \varphi(x)
$$

and $h:[a, \infty) \times \mathbb{C} \rightarrow \mathbb{C}$ is a continuous function which fulfils the condition

$$
|h(x, u(x))-h(x, v(x))| \leq \mu(x)|u(x)-v(x)|
$$

for all $x \in[a, \infty)$ and $u, v \in C_{b}([a, \infty))$.

If $y \in C_{b}([a, \infty))$ is such that

$$
\left|y(x)-p(x)-f(x, y(x)) \int_{a}^{x} g(x, \tau) h(\tau, y(\tau)) d \tau\right| \leq \varphi(x), \quad x \in[a, \infty),
$$

and $M \beta<1$, then there is a unique function $y_{0} \in C_{b}([a, \infty))$ such that

$$
y_{0}(x)=p(x)+f\left(x, y_{0}(x)\right) \int_{a}^{x} g(x, \tau) h\left(\tau, y_{0}(\tau)\right) d \tau
$$

and

$$
\left|y(x)-y_{0}(x)\right| \leq \frac{\varphi(x)}{1-M \beta}
$$

for all $x \in[a, \infty)$.

This means that under the above conditions, the corresponding Hammerstein integral equation has the HyersUlam-Rassias stability.

Proof. For any $n \in \mathbb{N}$, we will define $I_{n}=[a, a+n]$. By Theorem 3 , there exists a unique bounded continuous function $y_{0, n}: I_{n} \rightarrow \mathbb{C}$ such that

$$
y_{0, n}(x)=p(x)+f\left(x, y_{0, n}(x)\right) \int_{a}^{x} g(x, \tau) h\left(\tau, y_{0, n}(\tau)\right) d \tau
$$

and

$$
\left|y(x)-y_{0, n}(x)\right| \leq \frac{\varphi(x)}{1-M \beta}
$$


for all $x \in I_{n}$. The uniqueness of $y_{0, n}$ implies that if $x \in I_{n}$ then

$$
y_{0, n}(x)=y_{0, n+1}(x)=y_{0, n+2}(x)=\cdots
$$

For any $x \in[a, \infty)$, let us define $n(x) \in \mathbb{N}$ as $n(x)=\min \left\{n \in \mathbb{N} \mid x \in I_{n}\right\}$. We also define a function $y_{0}:[a, \infty) \rightarrow \mathbb{C}$ by

$$
y_{0}(x)=y_{0, n(x)}(x)
$$

For any $x_{1} \in[a, \infty)$, let $n_{1}=n\left(x_{1}\right)$. Then $x_{1} \in$ Int $I_{n_{1}+1}$ and there exists an $\epsilon>0$ such that $y_{0}(x)=y_{0, n_{1}+1}(x)$ for all $x \in\left(x_{1}-\epsilon, x_{1}+\epsilon\right)$. By Theorem 3, $y_{0, n_{1}+1}$ is continuous at $x_{1}$, and so it is $y_{0}$.

Now, we will prove that $y_{0}$ satisfies

$$
y_{0}(x)=p(x)+f\left(x, y_{0}(x)\right) \int_{a}^{x} g(x, \tau) h\left(\tau, y_{0}(\tau)\right) d \tau
$$

and

$$
\left|y(x)-y_{0}(x)\right| \leq \frac{\varphi(x)}{1-M \beta}
$$

for all $x \in[a, \infty)$. For an arbitrary $x \in[a, \infty)$ we chose $n(x)$ such that $x \in I_{n(x)}$. By (11) and (14), we have

$$
\begin{aligned}
y_{0}(x)=y_{0, n(x)}(x) & =p(x)+f\left(x, y_{0, n(x)}(x)\right) \int_{a}^{x} g(x, \tau) h\left(\tau, y_{0, n(x)}(\tau)\right) d \tau \\
& =p(x)+f\left(x, y_{0}(x)\right) \int_{a}^{x} g(x, \tau) h\left(\tau, y_{0}(\tau)\right) d \tau .
\end{aligned}
$$

Note that $n(\tau) \leq n(x)$, for any $\tau \in I_{n(x)}$, and it follows from (13) that $y_{0}(\tau)=y_{0, n(\tau)}(\tau)=y_{0, n(x)}(\tau)$, so, the last equality in (16) holds.

To prove (15), by (14) and (12), we have that for all $x \in[a, \infty)$,

$$
\left|y(x)-y_{0}(x)\right|=\left|y(x)-y_{0, n(x)}(x)\right| \leq \frac{\varphi(x)}{1-M \beta} .
$$

Finally, we will prove the uniqueness of $y_{0}$. Let us consider another bounded continuous function $y_{1}$ which satisfies (9) and (10), for all $x \in[a, \infty)$. By the uniqueness of the solution on $I_{n(x)}$ for any $n(x) \in \mathbb{N}$ we have that $y_{0 \mid I_{n(x)}}=y_{0, n(x)}$ and $y_{1_{\mid I_{n(x)}}}$ satisfies (9) and (10) for all $x \in I_{n(x)}$, so

$$
y_{0}(x)=\left.y_{0}\right|_{I_{n(x)}}(x)=\left.y_{1}\right|_{I_{n(x)}}(x)=y_{1}(x)
$$

Remark 6 With the necessary adaptations, Theorem 5 also holds true for infinite intervals $(-\infty, b]$, with $b \in \mathbb{R}$, as well as for $(-\infty, \infty)$.

\section{EXAMPLES}

In this section we will present some illustrative examples.

\section{First Example}

For continuous functions $y:\left[\frac{\pi}{12}, \frac{\pi}{6}\right] \rightarrow[-1,1]$, let us consider the integral equation

$$
y(x)=\sin (x)+\frac{x^{2}}{x+1}\left(\tan (x)-x-2+\sqrt{3}+\frac{\pi}{12}\right)+\frac{\arcsin (y(x))}{x+1} \int_{\frac{\pi}{12}}^{x}\left(-x \sin (\tau) \frac{y(\tau)}{\cos ^{2}(\tau)}\right) d \tau, \quad x \in\left[\frac{\pi}{12}, \frac{\pi}{6}\right],
$$

as well as the continuous function $\mu:\left[\frac{\pi}{12}, \frac{\pi}{6}\right] \rightarrow[0, \infty)$ such that $\mu(x)=\frac{1}{x}$. 
We realize that all the conditions of Theorem 3 are here satisfied. In fact, $p:\left[\frac{\pi}{12}, \frac{\pi}{6}\right] \rightarrow \mathbb{C}$ given by

$$
p(x)=\sin (x)+\frac{x^{2}}{x+1}\left(\tan (x)-x-2+\sqrt{3}+\frac{\pi}{12}\right)
$$

is a continuous function; $f:\left[\frac{\pi}{12}, \frac{\pi}{6}\right] \times[-1,1] \rightarrow \mathbb{C}$ such that $f(x, y(x))=\frac{\arcsin (y(x))}{x+1}$ is a continuous function which fulfills

$$
\sup _{x \in\left[\frac{\pi}{12}, \frac{\pi}{6}\right]}|f(x, y(x))|=\sup _{x \in\left[\frac{\pi}{12}, \frac{\pi}{6}\right]}\left|\frac{\arcsin (y(x))}{x+1}\right| \leq \frac{6 \pi}{\pi+12}=M
$$

the kernel $g:\left[\frac{\pi}{12}, \frac{\pi}{6}\right] \times\left[\frac{\pi}{12}, \frac{\pi}{6}\right] \rightarrow \mathbb{C}$ defined by $g(x, \tau)=-x \sin (\tau)$ is also continuous; there exists $\beta \in[0,1)$ such that

$$
\int_{\frac{\pi}{12}}^{x}|g(x, \tau)| \mu(\tau) \sigma(\tau) d \tau=\int_{\frac{\pi}{12}}^{x}|-x \sin (\tau)| \frac{1}{\tau} \tau d \tau=x\left(-\cos (x)+\cos \left(\frac{\pi}{12}\right)\right) \leq\left(\cos \left(\frac{\pi}{12}\right)-\cos \left(\frac{\pi}{6}\right)\right) x=\beta \sigma(x),
$$

where $\sigma:\left[\frac{\pi}{12}, \frac{\pi}{6}\right] \rightarrow(0, \infty)$ is the non-decreasing continuous function $\sigma(x)=x ; h:\left[\frac{\pi}{12}, \frac{\pi}{6}\right] \times[-1,1] \rightarrow \mathbb{C}$ such that $h(\tau, y(\tau))=\frac{y(\tau)}{\cos ^{2}(\tau)}$ is a continuous function which fulfils the condition

$$
\begin{aligned}
& |h(x, u(x))-h(x, v(x))|=\left|\frac{u(x)}{\cos ^{2}(x)}-\frac{v(x)}{\cos ^{2}(x)}\right| \leq \frac{1}{x}|u(x)-v(x)|=\mu(x)|u(x)-v(x)|, \quad x \in\left[\frac{\pi}{12}, \frac{\pi}{6}\right] . \\
& \text { If we choose } y(x)=\frac{\sin (x)}{0.6} \text {, it follows } \\
& \left|y(x)-p(x)-f(x, y(x)) \int_{0}^{x} g(x, \tau) h(\tau, y(\tau)) d \tau\right| \\
& =\left|\frac{2}{3} \sin (x)-\left[\frac{x^{2}}{x+1}-\frac{x}{(x+1) 0.6} \arcsin \left(\frac{\sin (x)}{0.6}\right)\right]\left(\tan (x)-x-2+\sqrt{3}+\frac{\pi}{12}\right)\right| \leq x=\sigma(x), \quad x \in\left[\frac{\pi}{12}, \frac{\pi}{6}\right] .
\end{aligned}
$$

Therefore, this exhibits the Hyers-Ulam-Rassias stability of the Hammerstein integral equation (17). Moreover, by using the exact solution $y_{0}(x)=\sin (x)$ and $M \beta=\frac{6 \pi}{\pi+12}\left(\cos \left(\frac{\pi}{12}\right)-\cos \left(\frac{\pi}{6}\right)\right) \simeq 0.124364632<1$, we realize that

$$
\left|y(x)-y_{0}(x)\right|=\left|\frac{\sin (x)}{0.6}-\sin (x)\right| \leq \frac{\sigma(x)}{1-M \beta}, \quad x \in\left[\frac{\pi}{12}, \frac{\pi}{6}\right] .
$$

\section{Second Example}

Let us now consider the integral equation

$$
y(x)=x-\frac{x}{2} \ln \left(\frac{x+1}{2}\right)+\frac{x y(x)}{2} \int_{1}^{x} \frac{1}{x+\tau} \frac{y(\tau)}{\tau^{2}} d \tau, \quad x \in[1,2],
$$

and the continuous given function $\mu:[1,2] \rightarrow[0, \infty)$ such that $\mu(x)=\frac{1}{x}$. We know that the exact solution of this equation is $y_{0}(x)=x$. Again, we can see that all the conditions of Theorem 3 are satisfied. In fact, $p:[1,2] \rightarrow \mathbb{C}$ such that

$$
p(x)=x-\frac{x}{2} \ln \left(\frac{x+1}{2}\right)
$$

is a continuous function; $f:[1,2] \times \mathbb{C} \rightarrow \mathbb{C}$ such that $f(x, y(x))=\frac{x y(x)}{2}$ is a continuous function where $\sup _{x \in[1,2]}|f(x, y(x))| \leq 2=M$; the kernel $g:[1,2] \times[1,2] \rightarrow \mathbb{C}$, given by $g(x, \tau)=\frac{1}{x+\tau}$, is a continuous; there exists $\beta \in[0,1)$ such that

$$
\int_{1}^{x}|g(x, \tau)| \mu(\tau) \sigma(\tau) d \tau=\int_{1}^{x}\left|\frac{1}{x+\tau}\right| \frac{1}{\tau} \tau d \tau=\ln \frac{2 x}{x+1} \leq \ln \frac{4}{3} x=\beta \sigma(x),
$$


where $\sigma:[1,2] \rightarrow(0, \infty)$ is the non-decreasing continuous function $\sigma(x)=x$. Moreover, $h:[1,2] \times \mathbb{C} \rightarrow \mathbb{C}$ such that $h(\tau, y(\tau))=\frac{y(\tau)}{\tau^{2}}$ is a continuous function which fulfils the condition

$$
|h(x, u(x))-h(x, v(x))|=\left|\frac{u(x)}{x^{2}}-\frac{v(x)}{x^{2}}\right|=\frac{1}{x^{2}}|u(x)-v(x)| \leq \frac{1}{x}|u(x)-v(x)|=\mu(x)|u(x)-v(x)|,
$$

for all $x \in[1,2]$. If we choose $y(x)=\frac{x}{0.3}$, it follows,

$$
\left|y(x)-p(x)-f(x, y(x)) \int_{a}^{x} g(x, \tau) h(\tau, y(\tau)) d \tau\right|=\left|\frac{17}{6}-\frac{50}{9} \ln \frac{x+1}{2}\right| x \leq\left|\frac{17}{6}-\frac{50}{9} \ln \frac{3}{2}\right| x \leq x=\sigma(x), x \in[1,2] .
$$

This exhibits the Hyers-Ulam-Rassias stability of the Hammerstein integral equation (18). In addition, having in mind the exact solution $y_{0}(x)=x$ and $M \beta=2 \times \ln \frac{4}{3} \simeq 0.5753641449<1$, we have

$$
\left|y(x)-y_{0}(x)\right|=\left|\frac{x}{0.3}-x\right| \leq \frac{\sigma(x)}{1-M \beta}=\frac{x}{1-2 \times \ln \frac{4}{3}}, \quad x \in[1,2] .
$$

\section{Third Example}

Finally, we will consider the integral equation

$$
y(x)=\sin (x)\left(\tan (x)-x-1+\sqrt{3}+\frac{\pi}{12}\right)+\frac{y(x)}{x} \int_{\frac{\pi}{12}}^{x}\left(-x \sin (\tau) \frac{y(\tau)}{\cos ^{2}(\tau)}\right) d \tau, \quad x \in\left[\frac{\pi}{12}, \frac{\pi}{8}\right],
$$

and the continuous given function $\mu:\left[\frac{\pi}{12}, \frac{\pi}{8}\right] \rightarrow[0, \infty)$ such that $\mu(x)=\frac{1}{x}$. We know that the exact solution of that equation is $y_{0}(x)=\sin (x)$.

We can see that all the conditions of Theorem 3 are satisfied. Namely, $p:\left[\frac{\pi}{12}, \frac{\pi}{8}\right] \rightarrow \mathbb{C}$ such that

$$
p(x)=\sin (x)\left(\tan (x)-x-1+\sqrt{3}+\frac{\pi}{12}\right)
$$

is a continuous function; $f:\left[\frac{\pi}{12}, \frac{\pi}{8}\right] \times \mathbb{C} \rightarrow \mathbb{C}$ such that $f(x, y(x))=\frac{y(x)}{x}$ is a continuous function where

$$
\sup _{x \in\left[\frac{\pi}{12}, \frac{\pi}{8}\right]}|f(x, y(x))|=\sup _{x \in\left[\frac{\pi}{12}, \frac{\pi}{8}\right]}\left|\frac{\sin (x)}{x}\right| \leq \frac{3(\sqrt{6}-\sqrt{2})}{\pi}=M
$$

the kernel $g:\left[\frac{\pi}{12}, \frac{\pi}{8}\right] \times\left[\frac{\pi}{12}, \frac{\pi}{8}\right] \rightarrow \mathbb{C}$ such that $g(x, \tau)=-x \sin (\tau)$ is continuous; there exists $\beta \in[0,1)$ such that

$$
\int_{\frac{\pi}{12}}^{x}|g(x, \tau)| \mu(\tau) \sigma(\tau) d \tau=\int_{\frac{\pi}{12}}^{x}|-x \sin (\tau)| \frac{1}{\tau} \tau d \tau=x\left(\cos \left(\frac{\pi}{12}\right)-\cos (x)\right) \leq\left(\cos \left(\frac{\pi}{12}\right)-\cos \left(\frac{\pi}{8}\right)\right) x=\beta \sigma(x),
$$

where $\sigma:\left[\frac{\pi}{12}, \frac{\pi}{8}\right] \rightarrow(0, \infty)$ is the non-decreasing continuous function $\sigma(x)=x$. Moreover, $h:\left[\frac{\pi}{12}, \frac{\pi}{8}\right] \times \mathbb{C} \rightarrow \mathbb{C}$ such that $h(\tau, y(\tau))=\frac{y(\tau)}{\cos ^{2}(\tau)}$ is a continuous function which fulfils the condition

$$
|h(x, u(x))-h(x, v(x))|=\left|\frac{u(x)}{\cos ^{2}(x)}-\frac{v(x)}{\cos ^{2}(x)}\right| \leq \frac{1}{x}|u(x)-v(x)|=\mu(x)|u(x)-v(x)|, \quad x \in\left[\frac{\pi}{12}, \frac{\pi}{8}\right] .
$$

If we choose $y(x)=\frac{\sin (x)}{0.8}$, it follows,

$$
\begin{aligned}
& \left|y(x)-p(x)-f(x, y(x)) \int_{\frac{\pi}{12}}^{x} g(x, \tau) h(\tau, y(\tau)) d \tau\right| \\
& =\left|\sin (x)\left(\frac{5}{4}-\tan (x)+x+1-\sqrt{3}-\frac{\pi}{12}+\frac{25}{16}\left(\tan (x)-x-2+\sqrt{3}+\frac{\pi}{12}\right)\right)\right| \leq x=\sigma(x), \quad x \in\left[\frac{\pi}{12}, \frac{\pi}{8}\right] .
\end{aligned}
$$

This exhibits the Hyers-Ulam-Rassias stability of the Hammerstein integral equation (19). Additionally, taking into account the exact solution $y_{0}(x)=\sin (x)$ and $M \beta=\frac{3(\sqrt{6}-\sqrt{2})}{\pi} \times\left(\cos \left(\frac{\pi}{12}\right)-\cos \left(\frac{\pi}{8}\right)\right) \simeq 0.0415676358<1$, we have

$$
\left|y(x)-y_{0}(x)\right|=\left|\frac{\sin (x)}{0.8}-\sin (x)\right| \leq \frac{\sigma(x)}{1-M \beta}, \quad x \in\left[\frac{\pi}{12}, \frac{\pi}{8}\right] .
$$




\section{ACKNOWLEDGMENTS}

This work was supported in part by FCT-Portuguese Foundation for Science and Technology through the Center for Research and Development in Mathematics and Applications (CIDMA) of Universidade de Aveiro, within project UID/MAT/04106/2013, and through the Center of Mathematics and Applications of University of Beira Interior, within project UID/MAT/00212/2013.

\section{REFERENCES}

[1] T. A. Burton, Volterra Integral and Differential Equations (Elsevier, Amsterdam, 2005).

[2] L. Cădariu, L. Găvruţa and P. Găvruça, "Weighted space method for the stability of some nonlinear equations", Appl. Anal. Discrete Math. 6(1), 126-139 (2012).

[3] L. P. Castro and R. C. Guerra, "Hyers-Ulam-Rassias stability of Volterra integral equations within weighted spaces", Lib. Math. (N.S.) 33(2), 21-35 (2013).

[4] L. P. Castro and A. Ramos, "Hyers-Ulam and Hyers-Ulam-Rassias stability of Volterra integral equations with a delay", in Integral Methods in Science and Engineering, Vol. 1, edited by M. E. Perez (Birkhäuser, Boston, 2010), pp. 85-94.

[5] L. P. Castro and A. Ramos, "Hyers-Ulam-Rassias stability for a class of nonlinear Volterra integral equations", Banach J. Math. Anal. 3(1), 36-43 (2009).

[6] L. P. Castro and A. Ramos, "Hyers-Ulam stability for a class of Fredholm integral equations", in Mathematical Problems in Engineering Aerospace and Sciences ICNPAA 2010, Proceedings of the 8th International Conference of Mathematical Problems in Engineering, Aerospace and Science, edited by S. Sivasundaram (Cambridge Scientific Publishers, Cambridge, 2011), pp. 171-176.

[7] Y. J. Cho, C. Park, T. M. Rassias and R. Saadati, Stability of Functional Equations in Banach Algebras (Springer International Publishing, Switzerland, 2015).

[8] C. C. Tisdell and A. Zaidi, "Basic qualitative and quantitative results for solutions to nonlinear, dynamic equations on time scales with an application to economic modelling", Nonlinear Anal. 68(1), 3504-3524 (2008).

[9] C. Corduneanu, Principles of Differential and Integral Equations (American Mathematical Society, New York, 1988).

[10] W.-S. Du, “A generalization of Diaz-Margolis's fixed point theorem and its application to the stability of generalized Volterra integral equations", J. Inequal. Appl. 2015:407, 15 pp. (2015).

[11] G.-L. Forti, "Hyers-Ulam stability of functional equations in several variables", Aequationes Math. 50, 143190 (1995).

[12] G. Gripenberg, S.-O. Londen and O. Staffans, Volterra Integral and Functional Equations (Cambridge University Press, Cambridge, 1990).

[13] D. H. Hyers, "On the stability of linear functional equation", Proc. Natl. Acad. Sci. 27(4), 222-224 (1941).

[14] D. H. Hyers, G. Isac and Th. M. Rassias, Stability of Functional Equations in Several Variables (Birkhäuser, Basel, 1998).

[15] S.-M. Jung, "A fixed point approach to the stability of an integral equation related to the wave equation", Abstr. Appl. Anal. 2013, Article ID 612576, 4 pp. (2013).

[16] S.-M. Jung, "A fixed point approach to the stability of a Volterra integral equation", Fixed Point Theory Appl. 2007, Article ID 57064, 9 pp. (2007).

[17] S.-M. Jung, Hyers-Ulam-Rassias Stability of Functional Equations in Mathematical Analysis (Hadronic Press, Palm Harbor, 2001).

[18] V. Lakshmikantham and M. R. M. Rao, Theory of Integro-differential Equations, Stability and Control: Theory, Methods and Applications (Gordon and Breach Science Publishers, Philadelphia, 1995).

[19] J. R. Morales and E. M. Rojas, "Hyers-Ulam and Hyers-Ulam-Rassias stability of nonlinear integral equations with delay", Int. J. Nonlinear Anal. Appl. 2(2), 1-6 (2011).

[20] D. Otrocol and V. Ilea, "Ulam stability for a delay differential equation”, Cent. Eur. J. Math 11(7), 1296-1303 (2013).

[21] D. Popa and I. Raşa, "On the best constant in Hyers-Ulam stability of some positive linear operators", J. Math. Anal. Appl. 412(1), 103-108 (2014).

[22] Th. M. Rassias, "On the stability of the linear mapping in Banach spaces", Proc. Amer. Math. Soc. 72, 297-300 (1978).

[23] S. Şevgina and H. Şevlib, "Stability of a nonlinear Volterra integro-differential equation via a fixed point approach”, J. Nonlinear Sci. Appl. 9(1), 200-207 (2016). 